\title{
A Deep Learning-Based Approach for Glomeruli Instance Segmentation from Multistained Renal Biopsy Pathologic Images
}

Lei Jiang, ${ }^{*}$ Wenkai Chen, ${ }^{\dagger}$ Bao Dong, ${ }^{\ddagger}$ Ke Mei, ${ }^{\dagger}$ Chuang Zhu, ${ }^{\dagger}$ Jun Liu, ${ }^{\dagger}$ Meishun Cai, ${ }^{\ddagger}$ Yu Yan, ${ }^{\ddagger}$ Gongwei Wang, ${ }^{\S}$ Li Zuo, ${ }^{\ddagger}$ and Hongxia Shi*

From the Electron Microscope Lab* and the Departments of Nephrology $y^{\ddagger}$ and Pathology, ${ }^{\S}$ Peking University People’s Hospital, Beijing; and the School of Information and Communication Engineering, ${ }^{\dagger}$ Beijing University of Posts and Telecommunications, Beijing, China

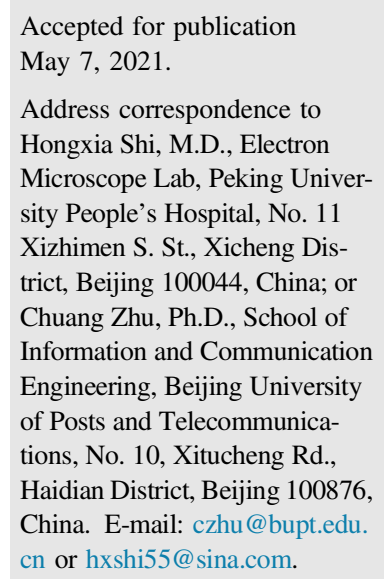

\begin{abstract}
Glomeruli instance segmentation from pathologic images is a fundamental step in the automatic analysis of renal biopsies. Glomerular histologic manifestations vary widely among diseases and cases, and several special staining methods are necessary for pathologic diagnosis. A robust model is needed to segment and classify glomeruli with different staining methods and apply in cases with various glomerular pathologic changes. Herein, pathologic images from renal biopsy slides stained with three basic special staining methods were used to build the data sets. The snapshot group included 1970 glomeruli from 516 patients, and the whole-slide image group included 8665 glomeruli from 148 patients. Cascade Mask region-based convolutional neural net architecture was trained to detect, classify, and segment glomeruli into three categories: i) GN, structural normal; ii) global sclerosis; and iii) glomerular with other lesions. In the snapshot group, total glomeruli, GN, global sclerosis, and glomerular with other lesions achieved an F1 score of $0.914,0.896,0.681$, and 0.756 , respectively, which were comparable with those in the whole-slide image group $(0.940,0.839,0.806$, and 0.753 , respectively). Among the three categories, GN achieved the best instance segmentation effect in both groups, as determined by average precision, average recall, F1 score, and Mask mean Intersection over Union. The present model segments and classifies multistained glomeruli with efficiency and robustness. It can be applied as the first step for more detailed glomerular histologic analysis. (Am J Pathol 2021, 191: 1431-1441; https://doi.org/10.1016/j.ajpath.2021.05.004)
\end{abstract}

Histologic evaluation of glomeruli from renal biopsy tissues plays a key role in the diagnosis of various kidney diseases, and important in making treatment strategies and predicting prognosis. ${ }^{1,2}$ Currently, histopathologic assessment of renal biopsies depends on manual judgment by pathologists. It is timeconsuming and has unsatisfactory reproducibility, especially in situations involving grading or scoring the histologic features, in spite of efforts to improve the definitions, criteria, and classification methods. ${ }^{3,4}$ In recent years, deep-learning methods, especially convolutional neural nets (CNNs), have been applied often in medical image analysis and have achieved good results. ${ }^{5-8}$ Renal biopsy pathology, as an important branch of pathology, has unique complexity in image analysis and urgently needs this new technology to improve the efficiency and accuracy of diagnosis.
Glomeruli extraction and recognition from pathologic images is a fundamental step in the automatic analysis of renal biopsies. Most of the recent work can generally be classified into two categories, object detection ${ }^{9-12}$ and semantic segmentation $^{13-19}$ (Table 1). For object detection, TemerinacOtt et $\mathrm{al}^{10}$ used CNN to get classification results from differently stained sections. They used it to improve glomeruli detection on one staining and achieved $10 \%$ to $20 \%$ higher F1 scores than those with the HOG detector. Bukowy et $\mathrm{al}^{12}$ used region-based convolutional neural net (R-CNN) and $\mathrm{CNN}$ for final

Supported by Peking University People's Hospital Research and Development Funds RDH2019-01 (L.J.).

L.J. and W.C. contributed equally to this work.

Disclosures: None declared. 
classification as glomerulus or background objects, and achieved an average precision and recall of $96.94 \%$ and $96.79 \%$, respectively. With the development of deep-learning methods, DeepLab-V2, ${ }^{17}$ SegNet, ${ }^{18}$ and U-Net ${ }^{18,19}$ have been used to deal with segmentation tasks in semantic segmentation. Object detection tasks can detect and classify glomeruli, but the boundary of glomeruli is unknown. Although semantic segmentation can determine the boundary, the glomeruli number cannot be calculated and different glomeruli cannot be distinguished in the same image. Therefore, to overcome the above limitations, the current method attempts to achieve the goal of instance segmentation. The algorithm of the Cascade Mask R$\mathrm{CNN}^{20}$ model was improved to better fit the data set so that sufficient image information can be acquired for further research.

Glomerular lesions can involve any part of a glomerulus, including endothelial cells, mesangial cells, podocytes, and mesangial matrix. ${ }^{21}$ In addition, the severity of lesions varies widely. Hence, the appearance of different glomeruli varies greatly. An ideal model should be able to identify and segment all types of glomeruli. Most of the existing models analyzing human kidney tissues for glomeruli detection and segmentation are based on a single disease, such as diabetic nephropathy, ${ }^{16}$ transplant biopsies, ${ }^{17}$ and $\operatorname{Ig}$ A nephropathy, ${ }^{19}$ except in case of Bueno et al ${ }^{18}$ who used 47 wholeslide images (WSIs) from AIDPATH kidney database.

In addition to hematoxylin-eosin staining, for light microscopy, a variety of histochemical stains, including periodic acid-Schiff (PAS), periodic acid-silver methenamine (PASM), and Masson trichrome stains are used to evaluate renal tissues. Each staining method has its unique value in elucidating specific histologic features and is essential for renal pathologic analysis. Both the variabilities of glomerular histologic manifestations and of staining methods increase the complexity of glomeruli detection and segmentation in pathologic images. Among the recent research analyzing human kidney tissues for glomeruli detection and segmentation, all results were obtained from PAS-stained sections. ${ }^{16-19}$ and there is no study that can segment the glomeruli from the images with multiple staining methods using a single model.

In this research, an algorithmic model with the following features was constructed: i) it can recognize and segment glomeruli with various histologic appearances from different diseases, ii) it has good robustness so that it can be applied to different special stained slides, and iii) it can make a preliminary classification of the glomeruli. For the above purposes, we present a deep-learning-based approach for the object extraction and three-classification of glomeruli.

\section{Materials and Methods}

\section{Tissue Samples}

Kidney biopsy samples from patients diagnosed with various kidney diseases from January 2018 to June 2019 in Department of Nephrology of Peking University People's Hospital (Beijing,
China) were collected. All biopsy samples were processed according to standard techniques for light microscopy, immunofluorescence, and electron microscopy. For light microscopy, formalin-fixed, paraffin-embedded tissues were cut into sections ( $2 \mu \mathrm{m}$ thick) and stained with one of the following techniques: hematoxylin-eosin, PAS, PASM, or Masson. The images from sections stained with PAS, PASM, and Masson were selected.

\section{Imaging}

The digital images used in this study were obtained from the two sources listed below.

\section{Snapshot Images from Image Library}

A total of 2 to 10 digital images were routinely captured in typical lesion areas from each case using Leica DFC550 digital camera (Singapore) attached to a Leica DM4000 B LED optical microscope (Weizla, Germany) and saved in the image library of our department. The average size of each image was approximately $1360 \times 1024 \times 3$ pixels, corresponding to a field of $2.176 \times 1.632 \mathrm{~mm}^{2}$, which resulted in a length scale of 0.85 $\mathrm{mm} /$ pixel.

Images from patients diagnosed with various kidney diseases from January 2018 to April 2019, with high technical staining quality and containing at least one glomerular area, were selected.

\section{Whole-Slide Images}

Slides were scanned at $40 \times$ using Precie 500B scanner (UNIC Technologies Inc., Beijing, China) for WSIs, with a $40 \times$ objective and numerical aperture $=0.8$; the resolution was $0.12 \mu \mathrm{m} /$ pixel for all acquired images.

Images from patients diagnosed with various kidney diseases from May 2019 to June 2019, with high technical staining quality, were selected.

\section{Glomerular Data Set Generation}

\section{Snapshot Images from Image Library}

In total, 1123 images from 516 patients, covering >30 pathologic types of kidney diseases, were collected (Tables 2 and 3). The diagnoses of the patients selected in this group are shown in Table 4. The total number of glomeruli was 1970 (PASM, 779; PAS, 492; and Masson, 699) (Table 5). The amplification multiples were $10 \times(65$ glomeruli), $20 \times$ (1482 glomeruli), and 40× (423 glomeruli).

\section{Whole-Slide Images}

A total of 348 WSIs were obtained from 148 patients with $>20$ pathologic types of kidney diseases (Tables 2 and 3). The total number of glomeruli was 8665 (PASM, 3248; PAS, 2525; and Masson, 2892) (Table 5). The diagnoses of the patients selected in this group are shown in Table 6.

Because of the high resolution of WSIs and the limited computing resources, the entire WSIs cannot be fed into the 
Table 1 Previous Research on Glomeruli Detection and Segmentation

\begin{tabular}{|c|c|c|c|c|c|}
\hline Source & Data/slide & Approach & Performance & $\begin{array}{l}\text { Source of renal tissue } \\
\text { images }\end{array}$ & Staining method \\
\hline \multicolumn{6}{|l|}{ Detection } \\
\hline Maree et $\mathrm{al}^{9}$ & Total: 200 & $\begin{array}{l}\text { Ellipsoidal shape }+ \text { decision } \\
\text { tree }\end{array}$ & $87 \% \mathrm{~F} 1$ & Human (renal biopsies) & Masson trichrome \\
\hline Temerinac-0tt et $\mathrm{al}^{10}$ & $\begin{array}{l}\text { Train: } 16 \\
\text { Test: } 4\end{array}$ & Mutual information + CNN & $66.38 \%-81.75 \%$ F1 & $\begin{array}{l}\text { 1. Human (nephrectomy } \\
\text { specimens) }\end{array}$ & $\begin{array}{c}\text { Jones H\&E, PAS, Sirius } \\
\text { Red, and immuno } \\
\text { stained for CD10 }\end{array}$ \\
\hline \multirow[t]{2}{*}{ Simon et $\mathrm{al}^{11}$} & $\begin{array}{l}\text { Train: } 25 \\
\text { Test: } 9\end{array}$ & Multiradial LBP + SVM & $\begin{array}{l}90.4 \% \text { Precision, } \\
76.7 \% \text { recall }\end{array}$ & $\begin{array}{l}\text { 1. A standard streptozocin } \\
\text { mouse model }\end{array}$ & $H \& E$ \\
\hline & & & & 2. Rats & $\begin{array}{l}\text { H\&E, PAS, Jones silver, } \\
\text { Gömöri trichrome, } \\
\text { and Congo Red }\end{array}$ \\
\hline Bukowy et $\mathrm{al}^{12}$ & $\begin{array}{l}\text { Train: } 72 \\
\text { Test: } 13\end{array}$ & Faster R-CNN & $\begin{array}{l}96.9 \% \text { Precision, } \\
96.8 \% \text { recall }\end{array}$ & $\begin{array}{l}\text { Rats of various genetic } \\
\text { backgrounds }\end{array}$ & Masson trichrome \\
\hline \multicolumn{6}{|l|}{ Segmentation } \\
\hline Kato et $\mathrm{al}^{13}$ & $\begin{array}{l}\text { Train:* } \\
\text { Test: } 20\end{array}$ & $\mathrm{~S}-\mathrm{HOG}+\mathrm{SVM}$ & $86.6 \% \mathrm{~F} 1$ & SD and SDT rats & $\begin{array}{l}\text { Immunostained for } \\
\text { desmin }\end{array}$ \\
\hline Sarder et $\mathrm{al}^{14}$ & $\begin{array}{l}\text { Train:* } \\
\text { Test: } 15\end{array}$ & Gabor filters & $87.8 \%$ Accuracy & $\begin{array}{l}\text { Normal healthy untreated } \\
\text { rats and mice }\end{array}$ & PAS and H\&E \\
\hline Sheehan et $\mathrm{al}^{15}$ & Total: 90 & AlexNet + SVM & $\begin{array}{l}92 \% \text { Recall, } \\
90 \% \text { specificity }\end{array}$ & $\begin{array}{l}\text { Mice with different } \\
\text { genotypes }\end{array}$ & PAS \\
\hline Zeng et $\mathrm{al}^{19}$ & $\begin{array}{l}\text { Test: } 22 \\
\text { Train: } 360\end{array}$ & $\begin{array}{l}\text { U-Net-SSIM + marked } \\
\text { watershed }\end{array}$ & $\begin{array}{l}\text { 93\% Specificity, } \\
\text { 93.1\% precision, } \\
94.0 \% \mathrm{~F} 1, \\
94.9 \% \text { recall, } \\
\text { 90.1\% Dice }\end{array}$ & $\begin{array}{l}\text { Human (patients with IgA } \\
\text { nephropathy) }\end{array}$ & PAS \\
\hline
\end{tabular}

*Not mentioned.

AIDPATH, Academia and Industry Collaboration for Digital Pathology; CNN, convolutional neural net; Dice, dice coefficient; DN, diabetes nephropathy; F1, F1 score; H\&E, hematoxylin-eosin; LBP, local binary patterns; PAS, periodic acid-Schiff; R-CNN, region-based CNN; SD, sprague-dawley; SDT, spontaneously diabetic torii; SVM, support vector machine; S-HOG, segmental histogram of oriented gradient.

model as input. So, the original WSIs were cropped into $2048 \times 2048$ pixel patches. In the cropping process, some glomeruli might be divided into two patches (incomplete cut). To avoid waste of data resources and to better use the data for getting a better model, the stride of the cutting window was three-fourths of the window's length. These images were then processed by script and checked manually to ensure that all irrelevant areas had been filtered out. The small patches were sent to the model, and the results were mapped back to WSIs, according to the cropped coordinates.

\section{Labeling Glomeruli}

Each image was labeled into one of the three categories:

i) GN: The glomeruli with completely open capillary tufts were considered structural normal (GN). This category included normal glomeruli, glomeruli with mild lesions (such as mild mesangial hypercellularity or mild mesangial matrix expansion), and glomeruli with thickened glomerular basement membrane without other lesions. 
Table 2 The Number and Distribution of Images and Patients in Two Groups of Data Set

\begin{tabular}{lrr}
\hline Data set & \multicolumn{1}{l}{ Snapshots } & \multicolumn{1}{l}{ WSIs } \\
\hline Training set & $1018 / 465(2.19)$ & $296 / 118(2.51)$ \\
Test set & $105 / 51(2.06)$ & $52 / 30(1.73)$ \\
Total & $1123 / 516(2.18)$ & $348 / 148(2.35)$ \\
\hline
\end{tabular}

Data are provided as number of images/number of patients (images/ patient).

WSI, whole-slide image.

ii) GS: The glomeruli with complete obliteration of the entire glomerular tuft and with loss of their normal structure were considered as global sclerosis (GS).

iii) GL: The glomeruli with lesions other than GS and leading to loss of any part of their structure were considered as glomerular with other lesions (GL). This category included segmental sclerosis, moderate to severe mesangial hypercellularity or expansion, crescents, and apparent endothelial proliferation.

Each lesion was defined according to the multicenter Nephrotic Syndrome Study Network digital pathology scoring system. ${ }^{22}$ Moderate to severe mesangial hypercellularity was defined as six or more mesangial cells/ mesangial area. ${ }^{23}$

Each image was independently reviewed and labeled by two nephropathologists (L.J. and G.W.). If there was disagreement, a third nephropathologist (expert) (B.D.) reviewed the image and discussed the labeling with the other two pathologists, to reach the final agreement.

\section{Data Set and Data Augmentation}

\section{Snapshot Images from Image Library}

The images were randomly split into two parts: 1803 glomeruli in the training set and 167 glomeruli in the test set. For the training set, data augmentation was used to increase the amount of training data and diversify the data distribution, so as to improve the robustness of the model. The specific transformation included random rotate 90 degrees, horizontal flip, vertical flip, transpose, and random crop. Thus, the final training set included a total of 10,818 glomeruli (Table 5).

Table 3 The Number and Distribution of WSIs and Snapshot Images With Each Stain

\begin{tabular}{lrrcr}
\hline Variable & PASM & PAS & Masson & \multicolumn{1}{c}{ All } \\
\hline Snapshots & & & & \\
$\quad$ Training set & 382 & 279 & 354 & 1018 \\
$\quad$ Test set & 39 & 22 & 44 & 105 \\
WSIs & & & & \\
$\quad$ Training set & 107 & 93 & 96 & 296 \\
$\quad$ Test set & 17 & 17 & 18 & 52 \\
\hline
\end{tabular}

Masson, Masson trichrome stain; PAS, periodic acid-Schiff; PASM, periodic acid-silver methenamine; WSI, whole-slide image.
Table 4 Diagnoses of the Patients in the Snapshots Group

\begin{tabular}{|c|c|c|}
\hline Diagnosis & $\begin{array}{l}\text { Patients, } \\
N\end{array}$ & $\begin{array}{l}\text { Patients, } \\
\%\end{array}$ \\
\hline IgA nephropathy & 145 & 28.10 \\
\hline Atypical membranous nephropathy & 121 & 23.45 \\
\hline Diabetic nephropathy & 47 & 9.11 \\
\hline Lupus nephritis & 26 & 5.04 \\
\hline Membranous nephropathy & 22 & 4.26 \\
\hline Focal and segmental glomerulosclerosis & 18 & 3.49 \\
\hline Henoch-Schonlein purpura nephritis & 16 & 3.10 \\
\hline Amyloidosis & 13 & 2.52 \\
\hline $\begin{array}{l}\text { ANCA-associated small-vessel } \\
\text { glomerulonephritis }\end{array}$ & 12 & 2.33 \\
\hline Minimal change disease & 9 & 1.74 \\
\hline Hepatitis B-associated glomerulonephritis & 7 & 1.36 \\
\hline IgA nephropathy + diabetic nephropathy* & 7 & 1.36 \\
\hline Subacute tubulointerstitial nephritis & 8 & 1.55 \\
\hline Crescentic glomerulonephritis & 6 & 1.16 \\
\hline Obesity-related glomerulopathy & 4 & 0.78 \\
\hline $\begin{array}{l}\text { Mild mesangial proliferative } \\
\text { glomerulopathy }\end{array}$ & 4 & 0.78 \\
\hline Ischemic renal injury & 4 & 0.78 \\
\hline Acute tubular injury & 4 & 0.78 \\
\hline Acute tubular necrosis & 4 & 0.78 \\
\hline Malignant hypertensive renal injury & 3 & 0.58 \\
\hline Focal proliferative glomerulonephritis & 3 & 0.58 \\
\hline $\begin{array}{l}\text { Atypical membranous nephropathy }+ \\
\text { diabetic nephropathy* }\end{array}$ & 3 & 0.58 \\
\hline $\begin{array}{l}\text { IgA nephropathy }+ \text { malignant } \\
\text { hypertensive renal injury* }\end{array}$ & 3 & 0.58 \\
\hline $\begin{array}{l}\text { IgA nephropathy }+ \text { membranous } \\
\text { nephropathy* }\end{array}$ & 3 & 0.58 \\
\hline Membranoproliferative glomerulonephritis & 3 & 0.58 \\
\hline Interstitial infiltration of leukemia cells & 2 & 0.39 \\
\hline Cast nephropathy & 2 & 0.39 \\
\hline $\begin{array}{l}\text { Minimal change disease }+ \text { ischemic renal } \\
\text { injury* }\end{array}$ & 2 & 0.39 \\
\hline $\begin{array}{l}\text { Endocapillary } \\
\text { proliferative glomerulonephritis }\end{array}$ & 3 & 0.58 \\
\hline Amyloidosis + membranous nephropathy* & 1 & 0.19 \\
\hline Light chain proximal tubulopathy & 1 & 0.19 \\
\hline Renocortical necrosis & 1 & 0.19 \\
\hline Lipoprotein glomerulopathy & 1 & 0.19 \\
\hline Chronic tubulointersitial nephritis & 1 & 0.19 \\
\hline Fabry disease & 1 & 0.19 \\
\hline $\begin{array}{l}\text { IgA nephropathy + ANCA- } \\
\text { associated small- } \\
\text { vessel glomerulonephritis* }\end{array}$ & 1 & 0.19 \\
\hline $\begin{array}{l}\text { IgA nephropathy }+ \text { minimal change } \\
\text { disease }^{*}\end{array}$ & 1 & 0.19 \\
\hline $\begin{array}{l}\text { Lupus nephritis }+ \text { thrombotic } \\
\text { microangiopathy* }\end{array}$ & 1 & 0.19 \\
\hline $\begin{array}{l}\text { Membranous nephropathy }+ \text { acute tubular } \\
\text { injury* }\end{array}$ & 1 & 0.19 \\
\hline Thin basement membrane nephropathy & 1 & 0.19 \\
\hline Thrombotic microangiopathy & 1 & 0.19 \\
\hline Total & 516 & 100.00 \\
\hline
\end{tabular}

*Compound diseases.

ANCA, anti-neutrophil cytoplasmic antibody. 
Table 5 The Number and Distribution of Glomeruli in Two Groups of Data Set

\begin{tabular}{lrrrr}
\hline Variable & GN & GL & GS & \multicolumn{1}{c}{ All } \\
\hline Snapshots & & & & \\
$\quad$ Original training set & 865 & 721 & 217 & 1803 \\
$\quad \begin{array}{l}\text { Training set after data } \\
\quad \text { enhancement }\end{array}$ & 5190 & 4326 & 1302 & 10,818 \\
$\quad \begin{array}{l}\text { Test set } \\
\text { WSIs } \\
\quad \text { Original training set }\end{array}$ & 81 & 65 & 21 & 167 \\
$\quad \begin{array}{l}\text { Training set after data } \\
\quad \text { enhancement }\end{array}$ & 3848 & 2258 & 1087 & 7193 \\
$\quad$ Test set & & 12,548 & 6516 & 43,152 \\
\hline
\end{tabular}

$\mathrm{GL}$, glomerulus with abnormal structure; GN, glomerulus with basically normal structure; GS, global sclerosis; WSI, whole-slide image.

Whole-Slide Images

Similar to the snapshot images, cropped WSIs were randomly split into two parts: 7193 in the training set and 1472 in the test set. After data augmentation, the final training set included a total of 43,152 glomeruli (Table 5).

Table 6 Diagnoses of the Patients in the WSI Group

\begin{tabular}{|c|c|c|}
\hline Diagnosis & Patients, $N$ & Patients, $\%$ \\
\hline IgA nephropathy & 75 & 50.68 \\
\hline Atypical membranous nephropathy & 22 & 14.86 \\
\hline Lupus nephritis & 7 & 4.73 \\
\hline Diabetic nephropathy & 6 & 4.05 \\
\hline $\begin{array}{l}\text { Hepatitis B-associated } \\
\text { glomerulonephritis }\end{array}$ & 5 & 3.38 \\
\hline Minimal change disease & 5 & 3.38 \\
\hline $\begin{array}{l}\text { Mild mesangial proliferative } \\
\text { glomerulopathy }\end{array}$ & 4 & 2.70 \\
\hline Focal and segmental glomerulosclerosis & 4 & 2.70 \\
\hline Membranous nephropathy & 4 & 2.70 \\
\hline $\begin{array}{l}\text { IgA nephropathy + diabetic } \\
\text { nephropathy* }\end{array}$ & 3 & 2.03 \\
\hline $\begin{array}{l}\text { Endocapillary } \\
\text { proliferative glomerulonephritis }\end{array}$ & 2 & 1.35 \\
\hline Thrombotic microangiopathy & 2 & 1.35 \\
\hline Henoch-Schonlein purpura nephritis & 2 & 1.35 \\
\hline $\begin{array}{l}\text { Membranoproliferative } \\
\text { glomerulonephritis }\end{array}$ & 1 & 0.68 \\
\hline Amyloidosis & 1 & 0.68 \\
\hline $\begin{array}{l}\text { ANCA-associated small-vessel } \\
\text { glomerulonephritis }\end{array}$ & 1 & 0.68 \\
\hline $\begin{array}{l}\text { Focal and segmental glomerulosclerosis } \\
\quad+\text { acute tubular injury }{ }^{*}\end{array}$ & 1 & 0.68 \\
\hline $\begin{array}{l}\text { IgA nephropathy }+ \text { membranous } \\
\text { nephropathy* }\end{array}$ & 1 & 0.68 \\
\hline $\begin{array}{l}\text { IgA nephropathy }+ \text { obesity-related } \\
\text { glomerulopathy }\end{array}$ & 1 & 0.68 \\
\hline Subacute tubulointerstitial nephritis & 1 & 0.68 \\
\hline Total & 148 & 100.00 \\
\hline
\end{tabular}

${ }^{*}$ Compound diseases.

ANCA, anti-neutrophil cytoplasmic antibody; WSI, whole-slide image.
Table 7 Detection and Segmentation Performance Metrics of Snapshot Images

\begin{tabular}{lllll}
\hline Variable & AP & AR & $\begin{array}{l}\text { F1 } \\
\text { score }\end{array}$ & $\begin{array}{l}\text { Mask } \\
\text { mIoU }\end{array}$ \\
\hline PASM & & & & \\
GN & 1.000 & 0.914 & 0.955 & 0.833 \\
GL & 0.880 & 0.880 & 0.880 & 0.812 \\
GS & 0.800 & 0.667 & 0.727 & 0.692 \\
Detection of total glomeruli* & 0.983 & 0.924 & 0.953 & 0.946 \\
PAS & & & & \\
GN & 1.000 & 1.000 & 1.000 & 0.943 \\
GL & 0.750 & 0.600 & 0.667 & 0.692 \\
GS & 0.615 & 0.889 & 0.727 & 0.692 \\
Detection of total glomeruli* & 0.843 & 0.900 & 0.871 & 0.886 \\
Masson & & & & \\
GN & 0.769 & 0.857 & 0.811 & 0.707 \\
GL & 0.690 & 0.667 & 0.678 & 0.647 \\
GS & 0.444 & 0.800 & 0.571 & 0.459 \\
Detection of total glomeruli & 0.857 & 0.943 & 0.898 & 0.907 \\
All & & & & \\
GN & 0.890 & 0.901 & 0.896 & 0.790 \\
GL & 0.774 & 0.738 & 0.756 & 0.708 \\
GS & 0.593 & 0.800 & 0.681 & 0.638 \\
Detection of total glomeruli* & 0.901 & 0.928 & 0.914 & 0.914 \\
\hline
\end{tabular}

*Regardless of the classification.

$A P$, average precision; $A R$, average recall; $G L$, glomerulus with abnormal structure; GN, glomerulus with basically normal structure; GS, global sclerosis; Mask mIoU, mean intersection/union ratio of the segmentation results; Masson, Masson trichrome stain; PAS, periodic acid-Schiff; PASM, periodic acid-silver methenamine.

\section{Model Training for Glomerular Instance Segmentation}

The architectures of the model are shown in Figure 1. Cascade Mask R-CNN architecture, based on CNNs, was trained by using the glomeruli training set. The adopted Cascade Mask R$\mathrm{CNN}$, which is built by extending Cascade $\mathrm{R}-\mathrm{CNN}^{20}$ by adding a branch for predicting an object mask in every stage, can detect, classify, and segment three types of glomeruli. ResNet-50 was used as the backbone of the model. ${ }^{24} \mathrm{~B} 0$ is the region proposal network, ${ }^{25}$ and other component details in the architectures are the same as in the Mask R-CNN. ${ }^{26}$ Among them, the backbone

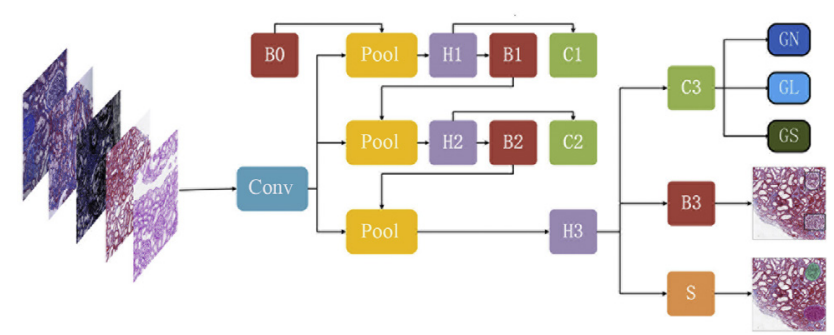

Figure 1 Architectures of the Cascade Mask region-based convolutional neural net. Bounding boxes of the glomeruli represent the detection results, which come after bounding box branch (B) 3, and the segmentation results come after segmentation branch $(\mathrm{S})$. $\mathrm{B} 0$, proposal in architecture; $\mathrm{C}$, classification branch; Conv, backbone convolutions; GL, glomerulus with abnormal structure; GN, glomerulus with normal structure; GS, global sclerosis; $\mathrm{H}$, network head; Pool, region-wise feature extraction. 

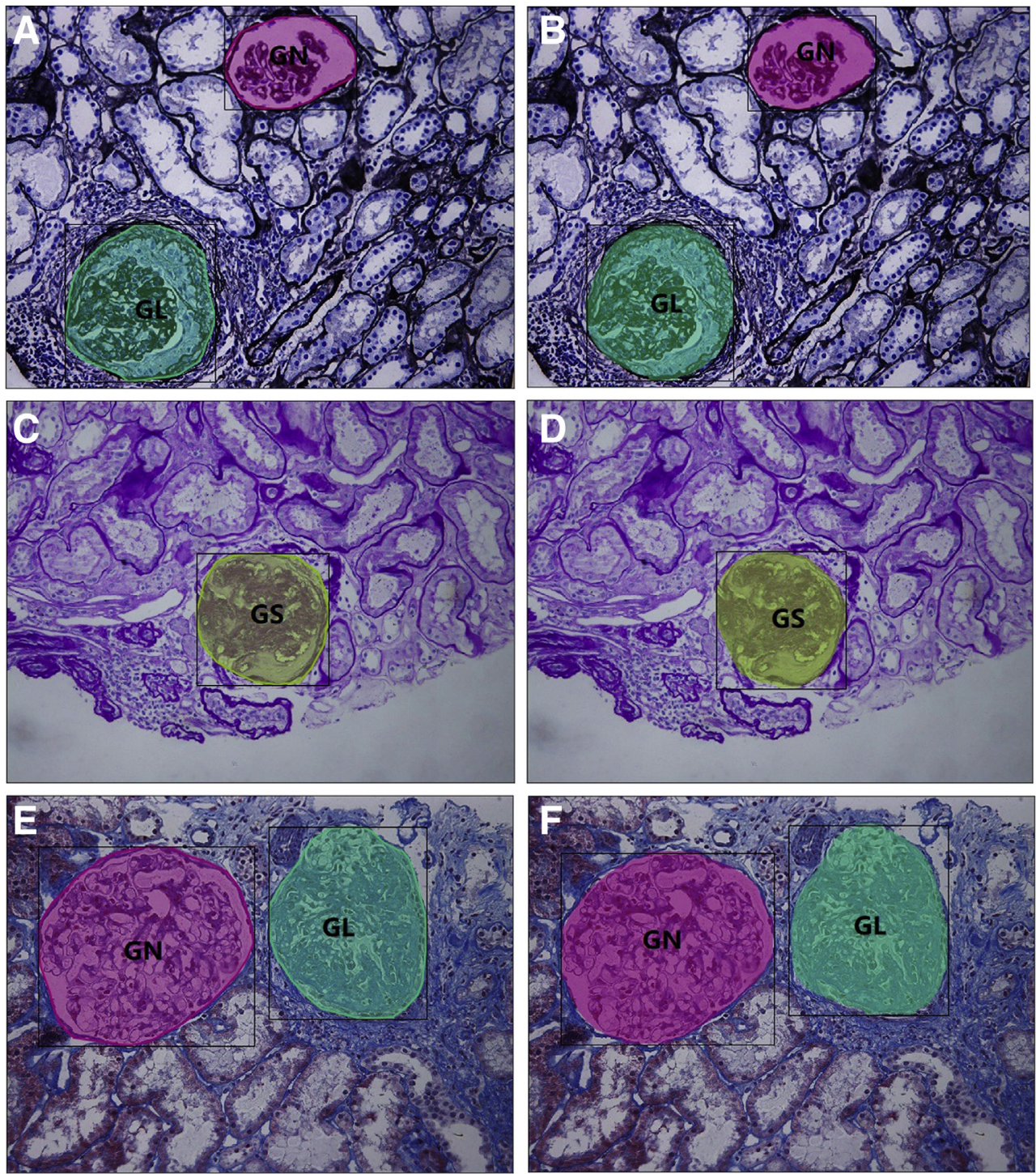

Figure 2 Segmentation and classification results of glomeruli in snapshot images. A, C, and E: Annotation by pathologists. B, D, and F: Artificial intelligence segmentation results. $\mathbf{A}-\mathbf{F}$ : The top $(\mathbf{A}$ and $\mathbf{B})$, middle $(\mathbf{C}$ and $\mathbf{D})$, and bottom $(\mathbf{E}$ and $\mathbf{F})$ panels were periodic acid-silver methenamine, periodic acid-Schiff, and Masson trichrome staining, respectively. GL, glomerulus with other abnormal structure; GN, glomerulus with normal structure; GS, global sclerosis.

consists of many convolution blocks that have the ability to extract image features; convolution blocks usually share some basic components, such as convolution layer and pooling layer. The convolutional layer is the fundamental component that is composed of various learnable kernels (filters). A pooling layer (usually applied after a convolutional layer) is used to compute feature maps condensed in a smaller representation with the goal of achieving some invariance. Region proposal network comprises two sibling fully connected layers-a box-regression layer and a box-classification layer follow convolution blocks. It can propose regions for use in subsequent model modules.

During the training procedure, part of the structure was modified to fit the data set and the hyperparameter was continuously changed after analyzing the results to get the model with the best performance. Specifically, the final classification layer was replaced from the network and retrained with the data set using the three output labels (GN, GL, and GS).

To adapt the model to different stainings, images with three different stains were combined to guide the model learning in the training stage, and the images were normalized to alleviate the domain differences between differently stained images. In the training process, some regular terms were added into the loss function, so that the model could avoid overfitting and had stronger robustness. Before being sent to the network for training, the images with different digital amplification were normalized to be under the same data distribution. The training process and the testing process used the same approach of normalization, so the model could be adapted to different resolutions.

The optimizer of choice for training was Stochastic Gradient Descent, with the learning rate of 0.005 , the momentum of 0.9 , weight decay of 0.0001 , and epoch of 12 . The learning rate 
dropped on epoch 8 and 11 . Smooth L1 was used as the loss function for the result of boundary box detection, and cross entropy was used as the loss function for classification and segmentation. The implementation is based on MMDetection, ${ }^{27}$ and code is available (https://github.com/bupt-aicz/glomeruli-instance-segmentation, last accessed April 1, 2021).

\section{Performance Metrics}

True positives (TPs) were defined as intersection over union (IoU) value $>0.75$, and the results with IoU value $<0.75$ were all judged to have failed detection.

Four metrics were used to evaluate the proposed method: average precision (AP) as the ratio of correctly predicting glomeruli and the sum of predicted TP and false-positive (FP) observations (whereby high precision is regarded to low FP rate); average recall (AR) as the ratio of correctly predicting glomeruli and the sum of predicted TP and false-negative (FN) observations (whereby high recall is regarded to low FN rate); and F1 score as the weighted average of precision and recall (whereby high F1 score is regarded to high precision and recall rates). Mask mean Intersection over Union (mIoU) was used as the mean IoU to evaluate the segmentation results; the areas of intersection and union were calculated by the segmentation part from ground truth (GT) and model prediction results $(\mathrm{P})$. AP, AR, and F1 score are detection metrics, and Mask mIoU is a segmentation metric.

The precision, recall, F1 score, and Mask IoU can be calculated as follows:

$$
\begin{gathered}
\text { Precision }=\frac{\mathrm{TP}}{\mathrm{TP}+\mathrm{FP}} \\
\text { Recall }=\frac{\mathrm{TP}}{\mathrm{TP}+\mathrm{FN}} \\
\mathrm{F} 1-\text { score }=\frac{2 * \text { Precision } * \text { Recall }}{\text { Precision }+ \text { Recall }} \\
\text { Mask IoU }=\frac{\mathrm{P} \cap \mathrm{GT}}{\mathrm{PUGT}}
\end{gathered}
$$

\section{Ethical Considerations}

This study was approved by the ethics committee of Peking University People's Hospital (2019PHB176-01).

\section{Results}

\section{Glomerular Instance Segmentation Using Snapshot Images}

Examples of the CNN pipeline applied to snap images are shown in Figure 2. Among the three categories, glomeruli with normal structure achieved the best instance segmentation

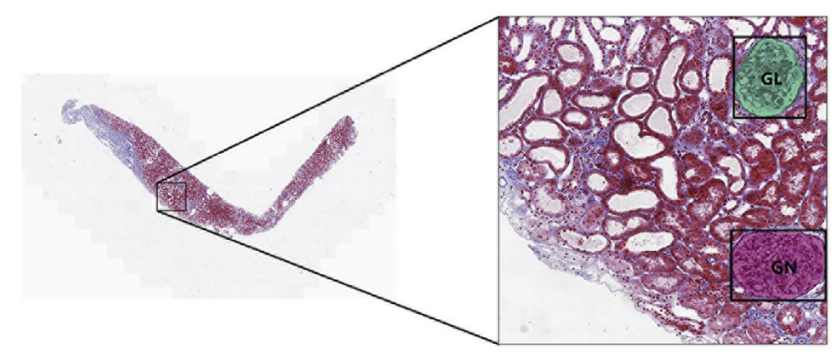

Figure 3 Example of glomerulus instance segmentation in whole-slide images. GL, glomerulus with other abnormal structure; GN, glomerulus with normal structure.

effect $(0.890,0.901,0.896$, and 0.790 for AP, AR, F1 score, and Mask mIoU, respectively), followed by glomeruli with abnormal structure $(0.774,0.738,0.756$, and 0.708 for AP, AR, F1 score, and Mask mIoU, respectively). Global sclerosis got the lowest scores $(0.593,0.800,0.681$, and 0.638 for AP, AR, F1 score, and Mask mIoU, respectively). AP, AR, F1 score, and Mask mIoU for total glomeruli ignoring the classification were also calculated, which were $0.901,0.928$, 0.914 , and 0.914 , respectively. The stain-wise performance was calculated, and the details are shown in Table 7. The orders of F1 scores were as follows: GN (PAS > PASM $>$ Masson), GL (PASM $>$ Masson $>$ PAS), GS (PASM = PAS $>$ Masson), and total (PASM $>$ Masson $>$ PAS).

\section{Glomerular Instance Segmentation Using WSIs}

To test the model's result, the WSI was first cropped into small patches, and then sent to the model, which finally mapped the results back to the WSI, according to the cropped coordinates. Mapping relation is shown in Figure 3.

Examples of the CNN pipeline applied to WSIs are shown in Figure 4. Among the three categories, glomerulus with basically normal structure achieved the best instance segmentation effect $(0.802,0.880,0.839$, and 0.702 for AP, AR, F1 score, and Mask mIoU, respectively), followed by global sclerosis (0.757, 0.862, 0.806, and 0.623 for AP, AR, F1 score, and Mask mIoU, respectively). The glomeruli with abnormal structure got the lowest scores $(0.866,0.666,0.753$, and 0.602 for AP, AR, F1 score, and Mask mIoU, respectively). AP, AR, F1 score, and Mask mIoU for total glomeruli ignoring the classification were also calculated, which were $0.957,0.924$, 0.940 , and 0.873 , respectively. The stain-wise performance was calculated, and the details are shown in Table 8 . The orders of F1 scores were as follows: GN (PASM $>$ PAS $>$ Masson), GL $($ PASM $>$ Masson $>$ PAS), GS (Masson > PASM > PAS), and total (PASM $>$ PAS $>$ Masson).

\section{Discussion}

For computer-aided automatic histologic assessment of a renal biopsy, the fundamental step is to extract the glomeruli with variable histologic features, as a human pathologist usually does in routine diagnosis works. To achieve this 

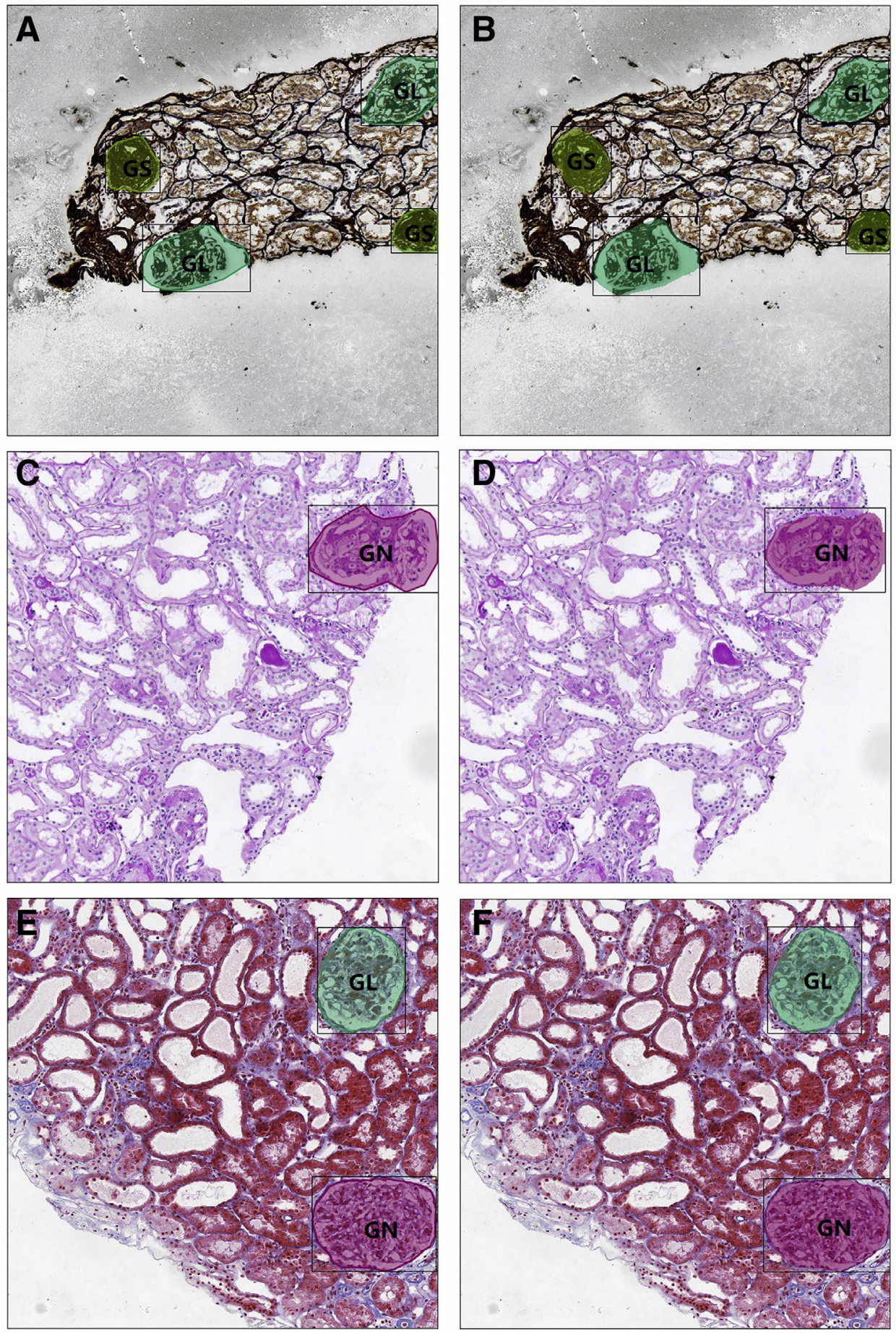

Figure 4 Segmentation and classification results of glomeruli in whole-slide images. A, C, and E: Annotation by pathologists. B, D, and F: Artificial intelligence segmentation results. $\mathbf{A}-\mathbf{F}$ : The top (A and $\mathbf{B})$, middle ( $\mathbf{C}$ and $\mathbf{D})$, and bottom (E and $\mathbf{F})$ panels were periodic acid-silver methenamine, periodic acid-Schiff, and Masson trichrome staining, respectively. GL, glomerulus with other abnormal structure; GN, glomerulus with normal structure; GS, global sclerosis.

goal, the data sets built in this study included $>30$ types of kidney diseases, covering most of the common kidney diseases, including combined situations, to develop a model that can detect both normal glomeruli and glomeruli with variable lesions with varying degrees of severity. So, the model presented in this study was designed to be consistent with the pathologist's daily workflow. This will lay a foundation for more detailed assessments of glomerular lesions in later research.

PASM, Masson, and PAS staining methods have been routinely used to show main structures and lesions of kidney tissues. To better meet the needs of future work, slides with all those three staining methods were contained in the data set to build a unified model with good robustness. Images 
Table 8 Detection and Segmentation Performance Metrics of WSIs

\begin{tabular}{lllll}
\hline Variable & AP & AR & $\begin{array}{l}\text { F1 } \\
\text { score }\end{array}$ & $\begin{array}{l}\text { Mask } \\
\text { mIoU }\end{array}$ \\
\hline PASM & & & & \\
GN & 0.847 & 0.908 & 0.876 & 0.742 \\
GL & 0.850 & 0.765 & 0.805 & 0.660 \\
GS & 0.761 & 0.836 & 0.797 & 0.621 \\
Detection of total glomeruli* & 0.947 & 0.961 & 0.954 & 0.881 \\
PAS & & & & \\
GN & 0.842 & 0.863 & 0.852 & 0.697 \\
GL & 0.823 & 0.614 & 0.703 & 0.544 \\
GS & 0.733 & 0.846 & 0.786 & 0.646 \\
Detection of total glomeruli* & 0.962 & 0.903 & 0.932 & 0.874 \\
Masson & & & & \\
GN & 0.683 & 0.852 & 0.758 & 0.616 \\
GL & 0.914 & 0.612 & 0.733 & 0.630 \\
GS & 0.767 & 0.917 & 0.853 & 0.616 \\
Detection of total glomeruli* & 0.966 & 0.894 & 0.929 & 0.856 \\
All & & & & \\
GN & 0.802 & 0.880 & 0.839 & 0.702 \\
GL & 0.866 & 0.666 & 0.753 & 0.602 \\
GS & 0.757 & 0.862 & 0.806 & 0.623 \\
Detection of total glomeruli* & 0.957 & 0.924 & 0.940 & 0.873 \\
\hline
\end{tabular}

*Regardless of the classification.

$A P$, average precision; $A R$, average recall; $G L$, glomerulus with abnormal structure; GN, glomerulus with basically normal structure; GS, global sclerosis; Mask mIoU, mean intersection/union ratio of the segmentation results; Masson, Masson trichrome stain; PAS, periodic acid-Schiff; PASM, periodic acid-silver methenamine; WSI, whole-slide image.

with different staining methods mean different statistical distributions of matrix values. On the whole, the result of PASM staining was the best, followed by PAS and Masson. For each classification, the performance orders were a little bit different. This might due to the different proportion of each stain. But because of the power of the algorithm, as long as enough data resources are obtained to train the model, it can perform well in images with each staining method. It will be simple and effective in use if a single model is applied with no need to consider the staining method. This study also confirmed the ability and adaptability of a deep-learning algorithm in the pathologic image analysis area.

There have been several studies to classify normal glomeruli and globally sclerosed glomeruli. ${ }^{18,28,29}$ In the present study, the glomeruli were classified into three categories, adding the GL tag to label the glomeruli with some lesions other than global sclerosis. Those lesions were selected because they are correlated with various glomerular diseases, and it is necessary to recognize them during pathologic analysis. Mild mesangial lesions, such as mild mesangial hypercellularity or mild mesangial matrix expansion, usually do not cause an obvious change of glomerular structure, so in the current step, they were labeled as GN and grouped with the normal glomeruli. In future research, it will be necessary to make more accurate annotations and design a more specific algorithm model to detect and segment each lesion.

Semantic segmentation networks have already been used in the classification and segmentation ${ }^{5,18,28-30}$ of glomeruli. But semantic segmentation is to classify all pixels in an image, so different glomeruli cannot be segmented separately. In clinical practice, counting the number of glomeruli is the first step of the pathologic analysis. Therefore, instance segmentation of glomeruli is necessary to meet the clinical needs. In this study, a Cascade Mask R-CNN ${ }^{20,24-26}$ algorithm model was trained for the instance segmentation of human glomeruli in routinely stained sections, using snapshot images and WSIs, respectively. The algorithm step of instance segmentation contained object detection and segmentation. The purpose of the object detection step is to locate the coordinates of glomeruli and classify their categories. IoU was used to describe the intersection with the real target border, and the IoU threshold was used as a reference to divide the positive samples and negative samples in training. Using Cascade Mask R-CNN, by means of cascade structure, different thresholds can be set so the contradiction of setting threshold value in a single module detection network can be avoided, and the accuracy of target detection can be greatly improved. For about segmentation step, the model segmented each detected glomerulus at the pixel level.

In the model building process, the strategy of the nonmaximum suppression (NMS) algorithm, which from $\mathrm{R}-\mathrm{CNN}^{31}$ was changed to suppress the redundant detection boxes. NMS algorithm calculates the IoU between each detection box. If some IoU values are too high, the algorithm will only save one detection box, which has the highest score. The general NMS algorithm only suppresses the results in the same class. The NMS algorithm was changed to suppress the

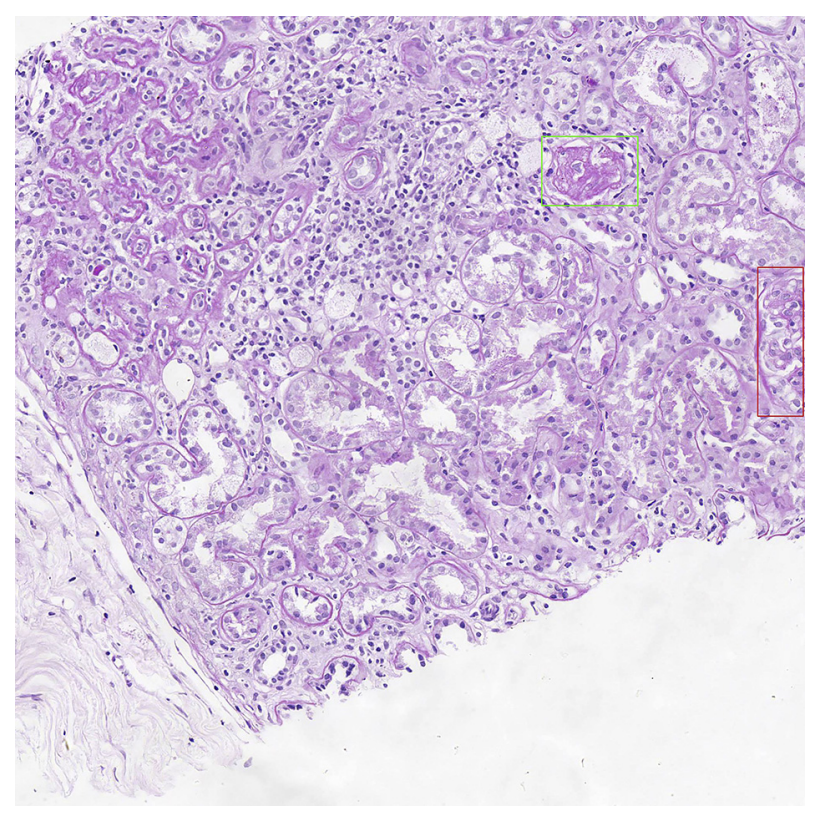

Figure 5 A failed detection case. Green bounding boxed area denotes successful detection, and red bounding boxed area denotes failed detection. 
results in all classes to prevent the generation of some incorrect results with the wrong classification. The improvement of NMS algorithm is effective for the data set.

Most of the failed detections were incomplete glomeruli located at the image boundary, as shown in Figure 5. Therefore, appropriate stride should be set during cutting patches to ensure that all glomeruli can be complete in at least one patch. In this way, when the results are mapped back to WSI, the detection results of glomeruli on the whole WSI will be better.

For some images of glomerular clusters, the segmentation results of the model output may overlap. To solve this problem, only the results with high confidence scores were reserved in the overlapping area.

For the classification of glomerular lesions, GN got the highest accuracy. The order of the performance metrics was just consistent with that of the sample size of each classification. To improve the accuracies of classifying GL and GS, more glomeruli with these tags need to be selected into the data set. Another reason might be that some glomeruli in GL and GS classification were seriously damaged and hard to be correctly detected from inflammatory interstitial tissue, such as a glomerular filled with crescent or a small sclerosis glomerulus without Bowman capsule. This also can be resolved by increasing the number of glomeruli with difficultly identified structures.

Although the process of biopsy digitization has been increasingly performed in diagnostic centers, there are still many smaller centers that do not have the digital scanner, and it is just these smaller centers that need more computer assistance to improve the accuracy of diagnosis. So, the images for training the model were from two sources: snapshot images from the image library and scanned WSIs. The snapshot images were taken from typical lesion areas of each case, so the proportion of pathologic glomeruli was relatively higher than that of glomeruli from WSIs. Although the total glomerular number was limited (only 1487 glomeruli), they belonged to $516 \mathrm{pa}$ tients and included $>30$ pathologic types of kidney diseases. All of those were conducive to the construction of an algorithm model for the recognition of glomeruli with varied lesions. The WSIs were from 148 patients, less than those of snapshot images, and they also contained fewer pathologic types of kidney diseases, whereas the total number of glomeruli was nearly four times that of the former. The performance metrics, which reflected overall accuracy of recognition, were comparable in two groups, especially when detecting total glomeruli, regardless of classification; AP, AR, and F1 score were $>0.9$. This might be explained that large sample size made up for the lack of diversity of lesions in the WSI group, to some extent. To build an ideal data set, both sample size and lesion diversity should be considered in future studies.

This study also has some limitations. First, as has been mentioned above, the number of glomeruli with seriously damaged structures should be increased to improve segmentation accuracy. Second, the abnormal glomeruli detected in this model only included lesions that obviously changed the glomerular structures, whereas the thickness of the basement membrane was not considered. It needs a more refined model to detect such lesions. Third, in terms of classification, the result of basically normal glomeruli is the best, and the other two categories need to be judged and modified by physicians on the basis of the automatic classification of the model. We are working on an application with an interactive window to help physicians adjust the classification as they apply the model, and the results can be used to further refine the model.

In conclusion, this study presents a model that can segment both normal glomeruli and glomeruli with various lesions, suitable for three kinds of special staining methods at the same time, and can detect and classify glomeruli into three types. In clinical practice, the present model can help physicians to calculate the number of glomeruli, quickly locate glomeruli in diagnosis, and lay the foundation for further analysis of the internal structures of glomeruli. Therefore, the current study can be regarded as the first step in the whole automation system. Images can be put into this part to get the bounding box and classification results, then cropped into smaller patches by bounding box value and sent to the next parts to analyze more detailed histologic information, such as mesangial hypercellularity and crescents.

\section{Acknowledgments}

We thank Xin Li and Chunying Shao for excellent technical assistance.

\section{Author Contributions}

L.J., C.Z., and J.L. designed the research. L.J., W.C., and K.M. analyzed and interpreted the data. L.J. and W.C. drafted the article. B.D., J.L., and G.W. provided intellectual content of critical importance to the work. C.Z., H.S., M.C., Y.Y., and L.Z. revised the article. C.Z. and H.S. approved the version to be published.

H.S. and C.Z. are the guarantors of this work and, as such, had full access to all of the data in the study and take responsibility for the integrity of the data and the accuracy of the data analysis.

\section{References}

1. Puelles VG, Bertram JF: Counting glomeruli and podocytes: rationale and methodologies. Curr Opin Nephrol Hypertens 2015, 24:224-230

2. Lees GE, Cianciolo RE, Clubb FJ: Renal biopsy and pathologic evaluation of glomerular disease. Top Companion Anim Med 2011, 26:143-153

3. Bajema IM, Wilhelmus S, Alpers CE, Bruijn JA, Colvin RB, Cook HT, D'Agati VD, Ferrario F, Haas M, Jennette JC, Joh K, Nast CC, Noel LH, Rijnink EC, Roberts ISD, Seshan SV, Sethi S, Fogo AB: Revision of the International Society of Nephrology/Renal Pathology Society classification for lupus nephritis: clarification of definitions, and modified National Institutes of Health activity and chronicity indices. Kidney Int 2018, 93:789-796 
4. Trimarchi H, Barratt J, Cattran DC, Cook HT, Coppo R, Haas M, Liu ZH, Roberts IS, Yuzawa Y, Zhang H, Feehally J; IgAN Classification Working Group of the International IgA Nephropathy Network and the Renal Pathology Society: Oxford classification of IgA nephropathy 2016: an update from the IgA Nephropathy Classification Working Group. Kidney Int 2017, 91:1014-1021

5. Gadermayr M, Dombrowski A-K, Klinkhammer BM, Boor P, Merhof D: CNN cascades for segmenting sparse objects in gigapixel whole slide images. Comput Med Imaging Graphics 2019, 71:40-48

6. Esteva A, Robicquet A, Ramsundar B, Kuleshov V, DePristo M, Chou K, Cui C, Corrado G, Thrun S, Dean J: A guide to deep learning in healthcare. Nat Med 2019, 25:24-29

7. Wang S, Yang DM, Rong R, Zhan X, Xiao G: Pathology image analysis using segmentation deep learning algorithms. Am J Pathol 2019, 189:1686-1698

8. Komura D, Ishikawa S: Machine learning approaches for pathologic diagnosis. Virchows Arch 2019, 475:131-138

9. Maree R, Dallongeville S, Olivo-Marin JC, Meas-Yedid V: An approach for detection of glomeruli in multisite digital pathology. IEEE International Symposium on Biomedical Imaging; 2016. pp. 1033-1036. IEEE

10. Temerinac-Ott M, Forestier G, Schmitz J, Hermsen M, Brasen JH, Feuerhake F, Wemmert C: Detection of glomeruli in renal pathology by mutual comparison of multiple staining modalities. 10th International Symposium on Image and Signal Processing and Analysis; 2017. pp. 19-24. Ljubljana, Slovenia

11. Simon O, Yacoub R, Jain S, Tomaszewski JE, Sarder P: Multi-radial LBP features as a tool for rapid glomerular detection and assessment in whole slide histopathology images. Sci Rep 2018, 8:2032

12. Bukowy JD, Dayton A, Cloutier D, Manis AD, Staruschenko A, Lombard JH, Solberg Woods LC, Beard DA, Cowley AW Jr: Regionbased convolutional neural nets for localization of glomeruli in trichromestained whole kidney sections. J Am Soc Nephrol 2018, 29:2081-2088

13. Kato T, Relator R, Ngouv H, Hirohashi Y, Takaki O, Kakimoto T, Okada K, Segmental HOG: new descriptor for glomerulus detection in kidney microscopy image. BMC Bioinformatics 2015, 16:1-16

14. Sarder P, Ginley B, Tomaszewski JE: Automated renal histopathology: digital extraction and quantification of renal pathology. SPIE Medical Imaging; 2016. San Diego, CA

15. Sheehan S, Mawe S, Cianciolo RE, Korstanje R, Mahoney JM: Detection and classification of novel renal histologic phenotypes using deep neural networks. Am J Pathol 2019, 189:1786-1796

16. Ginley B, Lutnick B, Jen K-Y, Fogo AB, Jain S, Rosenberg A, Walavalkar V, Wilding G, Tomaszewski JE, Yacoub R, Rossi GM, Sarder P: Computational segmentation and classification of diabetic glomerulosclerosis. J Am Soc Nephrol 2019, 30:1953-1967

17. Hermsen M, de Bel T, den Boer M, Steenbergen EJ, Kers J, Florquin S, Roelofs JJTH, Stegall MD, Alexander MP, Smith BH, Smeets B, Hilbrands LB, van der Laak JAWM: Deep learning-based histopathologic assessment of kidney tissue. J Am Soc Nephrol 2019, 30:1968-1979

18. Bueno G, Fernandez-Carrobles MM, Gonzalez-Lopez L, Deniz O: Glomerulosclerosis identification in whole slide images using semantic segmentation. Comput Methods Programs Biomed 2020, 184:105273

19. Zeng C, Nan Y, Xu F, Lei Q, Li F, Chen T, Liang S, Hou X, Lv B, Liang D, Luo W, Lv C, Li X, Xie G, Liu Z: Identification of glomerular lesions and intrinsic glomerular cell types in kidney diseases via deep learning. J Pathol 2020, 252:53-64

20. Cai Z, Vasconcelos N, Cascade R-CNN: High Quality Object Detection and Instance Segmentation. High Quality Object Detection and Instance Segmentation 2021, 43:1483-1498

21. Sethi S, Haas M, Markowitz GS, D'Agati VD, Rennke HG, Jennette JC, Bajema IM, Alpers CE, Chang A, Cornell LD, Cosio FG, Fogo AB, Glassock RJ, Hariharan S, Kambham N, Lager DJ, Leung N, Mengel M, Nath KA, Roberts IS, Rovin BH, Seshan SV, Smith RJH, Walker PD, Winearls CG, Appel GB, Alexander MP, Cattran DC, Casado CA, Cook HT, De Vriese AS, Radhakrishnan J, Racusen LC, Ronco P, Fervenza FC: Mayo Clinic/Renal Pathology Society consensus report on pathologic classification, diagnosis, and reporting of GN. J Am Soc Nephrol 2016, 27:1278-1287

22. Barisoni L, Troost JP, Nast C, Bagnasco S, Avila-Casado C, Hodgin J, Palmer M, Rosenberg A, Gasim A, Liensziewski C, Merlino L, Chien HP, Chang A, Meehan SM, Gaut J, Song P, Holzman L, Gibson D, Kretzler M, Gillespie BW, Hewitt SM: Reproducibility of the NEPTUNE descriptor-based scoring system on whole-slide images and histologic and ultrastructural digital images. Mod Pathol 2016, 29: 671-684

23. Working Group of the International Ig A Nephropathy Network and the Renal Pathology Society, Roberts IS, Cook HT, Troyanov S, Alpers CE, Amore A, Barratt J, Berthoux F, Bonsib S, Bruijn JA, Cattran DC, Coppo R, D'Agati V, D'Amico G, Emancipator S, Emma F, Feehally J, Ferrario F, Fervenza FC, Florquin S, Fogo A, Geddes CC, Groene HJ, Haas M, Herzenberg AM, Hill PA, Hogg RJ, Hsu SI, Jennette JC, Joh K, Julian BA, Kawamura T, Lai FM, Li LS, et al: The Oxford classification of IgA nephropathy: pathology definitions, correlations, and reproducibility. Kidney Int 2009, 76:546-556

24. He K, Zhang X, Ren S, Sun J: Deep residual learning for image recognition. IEEE Conference on Computer Vision \& Pattern Recognition; 2016. pp. 770-778. IEEE Computer Society

25. Ren S, He K, Girshick R, Sun J, Faster R-CNN: Towards real-time object detection with region proposal networks. IEEE Trans Pattern Anal Mach Intell 2016, 39:1137-1149

26. He K, Gkioxari G, Dollár P, Girshick R: Mask R-CNN. In 2017 IEEE International Conference on Computer Vision (ICCV). Venice, Italy, IEEE, 2017

27. Chen K, Wang J, Pang J, Cao Y, Xiong Y, Li X, Sun S, Feng W, Liu Z, Xu J: MMDetection: Open MMLab Detection Toolbox and Benchmark. arXiv 2019, 1906.07155v1

28. Marsh JN, Matlock MK, Kudose S, Liu TC, Stappenbeck TS, Gaut JP, Swamidass SJ: Deep learning global glomerulosclerosis in transplant kidney frozen sections. IEEE Trans Med Imaging 2018, 37:2718-2728

29. Kannan S, Morgan LA, Liang B, Cheung MG, Lin CQ, Mun D, Nader RG, Belghasem ME, Henderson JM, Francis JM, Chitalia VC, Kolachalama VB: Segmentation of glomeruli within trichrome images using deep learning. Kidney Int Rep 2019, 4:955-962

30. Barros GO, Navarro B, Duarte A, Dos-Santos WLC: PathoSpotter-K: a computational tool for the automatic identification of glomerular lesions in histological images of kidneys. Sci Rep 2017, 7:46769

31. Girshick R, Donahue J, Darrell T, Malik J: Rich feature hierarchies for accurate object detection and semantic segmentation. In 2014 IEEE Conference on Computer Vision and Pattern Recognition (CVPR). Columbus, OH, IEEE, 2014 